\title{
Anabases
}

ANABASES Traditions et réceptions de l'Antiquité

$26 \mid 2017$

Varia

\section{CURIOSA (2). In vino (vin/eau) veritas (very tasse) : drame de l'ivresse}

\section{Claude Aziza}

\section{(2) OpenEdition}

1 Journals

Édition électronique

URL : http://journals.openedition.org/anabases/6366

DOI : $10.4000 /$ anabases.6366

ISSN : 2256-9421

Éditeur

E.R.A.S.M.E.

\section{Édition imprimée}

Date de publication : 1 novembre 2017

Pagination : 189-191

ISSN : 1774-4296

\section{Référence électronique}

Claude Aziza, "CURIOSA (2). In vino (vin/eau) veritas (very tasse) : drame de l'ivresse », Anabases [En ligne], 26 | 2017, mis en ligne le 01 novembre 2020, consulté le 20 janvier 2021. URL : http:// journals.openedition.org/anabases/6366 ; DOI : https://doi.org/10.4000/anabases.6366 


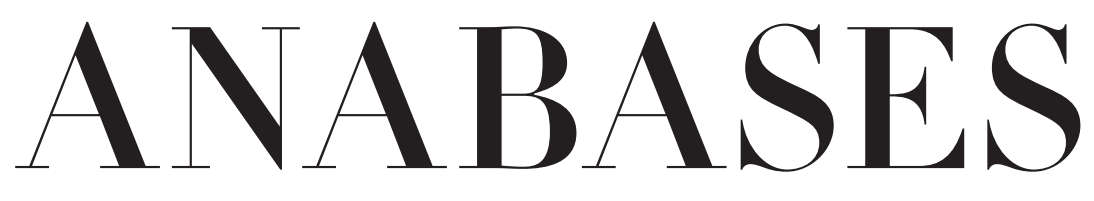

Traditions et Réceptions de l'Antiquité

$$
\begin{aligned}
& N \circ 26 \\
& 2017
\end{aligned}
$$

\section{E.R.A.S.M.E.}

Université Toulouse - Jean Jaurès 



\section{Sommaire}

$\mathrm{N}^{\circ} 26-2017$

\section{Historiographie et identités culturelles}

Jocelyne Peigney

La polysémie poétique d'anabase chez Saint-John Perse :

évocations, étymologie et botanique $\ldots \ldots \ldots \ldots \ldots \ldots \ldots \ldots \ldots \ldots$

Nicolas FAELLI

Les références antiques des fondateurs de l'Amérique française

au XVII siècle ................................. I9

Silvia Alaura

Setting the stage for Hittite studies in Victorian England:

practices and methods of the 1870 . . . . . . . . . . . . .

\section{Traditions du patrimoine antique}

“ Restituer Herculanum I. Des archives de fouilles aux restitutions 3D »

Alexandra Dardenay et Adeline Grand-Clément

Restituer Herculanum au XXI ${ }^{\mathrm{e}}$ siècle. L'apport des archives. . . . . . . . 55

Antonella Coralini

In situ e alibi, dallo scavo integrato alla cultura dell'abitare :

Vesuviana-DHER a Ercolano . . . . . . . . . . . . . . . . 67 67

Agnes Allroggen-Bedel

I documenti settecenteschi come strumenti per lo studio

degli scavi ercolanesi . . . . . . . . . . . . . . . Io3

James ANDREws

Revisiting the upper floors of the casa di Argo at Herculaneum . . . . . . II7 
Nicolas MonteIx

Les archives des fouilles d'Herculanum au XX $\mathrm{XX}^{\mathrm{e}}$ siècle :

analyse critique et pistes d'exploitation . . . . . . . . . . I43

\section{Archéologie des savoirs}

Yannick Le PAPE

L'inspiration et l'imprudence. Poésie de l'anticomanie

dans la critique d'art du second XIX ${ }^{\mathrm{e}}$ siècle . . . . . . . . . . . . . I57

\section{Actualités et débats}

Claude AzızA

Antiquités parallèles (7). La starlette des bandelettes . . . . . . . . . I77

Alexandra TrachseL

La réception d'Homère dans le domaine de la rhétorique $\ldots \ldots \ldots$. . . I83

Claude Aziza

Curiosa (2) In vino (vin/eau) veritas (very tasse) : drame de l'ivresse . . . . I89

\section{Lire, relire la bibliothèque des sciences de l'Antiquité}

Laurent OLIVIER

Pour en finir avec la race : Henri Hubert et l'anthroposociologie

À propos du commentaire de “L'Aryen, son rôle social », de Vacher de Lapouge $(\mathrm{r} 899) \ldots \ldots \ldots$

Henri Hubert

Compte rendu de Georges VACher de LAPouge, L'Aryen, son rôle social, L'Année sociologique, igoo, p. i45-146 . . . . . . . . . . . . . . . . . 203

\section{L'atelier de l'histoire : chantiers historiographiques}

L'Atelier des doctorants (coordonné par Adeline Grand-Clément) (14)

Francesco LoDÀ,

L'initiation des guerriers chez les peuples indo-européens.

Une enquête historiographique dans les pas de Georges Dumézil . . . 207

\section{Comptes rendus}

Benjamin Anderson et Robert G. Ousterhout

Palmyra I885. The Wolfe Expedition and the photographs

of John Henry Haynes (M. Sartre) . . . . . . . . . . . . . . . . . . . 2 215 
Mary R. BAchvarova

From Hittite to Homer. The Anatolian Background of Ancient Greek Epic (G. Hoffmann) . . . . . . . . . . . . . . . . .

Patrick Baker, Ronny Kaiser, Maike Priesterjahn, Johannes Helmrath (éd.) Portraying the Prince in the Renaissance. The Humanist Depiction of Rulers in Historiographical and Biographical Texts (G. Demelemestre). . 220

Brigitte BAKHоUсHE (éd.)

Science et exégèse. Les interprétations antiques et médiévales du récit biblique de la création des éléments (Genèse I, I-8) (F. P. BARONE) . . . . . . .

Laurence Baurain-Rebillard (éd.)

Héros grecs à travers le temps : autour de Persée, Thésée, Cadmos et Bellérophon (C. Giovénal) . . . . . . . . . . . . . . . .

Mark W. PAdiLla,

Classical myth in four films of Alfred Hitchcock . . . . . . . . . . . . . . .

Vivien Bessieres

Le Péplum et après? L’Antiquité gréco-romaine dans les récits contemporains (Cl. Aziza) . . . . . . . . . . . . . . . . . 225

Pierre Briant

Alexandre. Exégèse des lieux communs (É. Guillon) . . . . . . . . . . . . . 227

Paulo ButTi DE Lima

Il piacere delle Immagini. Un tema aristotelico nella riflessione moderna sull'arte (C. Noacco) . . . . . . . . . . . . . . . . . . .

Bénédicte Coudì̀re

La truelle et le phylactère. La proximité des images (M. Scapin) . . . . . . . 23I

Thorsten Fögen, Richard WARREN (éd.)

Graeco-Roman Antiquity and the Idea of Nationalism in the rgth Century, Case Studies (M. Champier) . . . . . . . . . . . . . . . ${ }_{232}$

Ronald H. Fritze

Egyptomania. A History of Fascination, Obsession and Fantasy

(Fabien Bièvre-Perrin) . . . . . . . . . . . . . . . . . . . . . 234

Joseph Geiger

Hellenism in the East. Studies on Greek Intellectuals in Palestine

(C. Bonnet). . . . . . . . . . . . . . . . . 236

Tiffany Jenkins

Keeping their marbles. How the treasures of the past ended up in museums... and why they should stay there (C. Valenti) . . . . . . . . . . 238 
Ayelet H. Lushkov

Magistracy and the Historiography of the Roman Republic.

Politics in Prose (C. Landrea) . . . . . . . . . . . . . . . . . . . . . . 240

Jean-Claude MüHLEthaler

Énée le mal-aimé. Du roman médiéval à la bande dessinée

(Fl. Bouchet) ........................ 24I

Laurent PERNoT

Epideictic Rhetoric, Questioning the Stakes of Ancient Praise

(C.Psilakis) ......................... 243

Priscian

Answers to King Khosroes of Persia (O. Gengler) . . . . . . . . . . . . . . 245

Paul Robertson

Paul's letters and contemporary Greco-Roman literature:

theorizing a new taxonomy (M. Cambron-Goulet) . . . . . . . . . . . . . . 247

Federico Santangelo (éd.)

Approaching the Roman Revolution. Sir Ronald Syme,

Papers on Republican History (C. Landrea) . . . . . . . . . . . . . . . . . . 249

Molly Swetnam-BurLand

Egypt in Italy. Visions of Egypt in Roman Imperial Culture

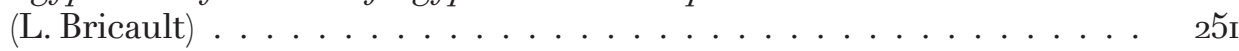

Helen VAN Noorden

Playing Hesiod. The 'Myth of the Races' in Classical Antiquity

(D. Bonanno)

Résumés . . . . . . . . . . . . . . . . . . . . . . . . . . . . . . . 257

Index .......................... . . 265 


\section{Actualités et débats}



Anabases 26 (2017), p. 189-191.

\section{CURIOSA (2) \\ In vino (vin/eau) veritas (very tasse) : drame de l'ivresse}

Claude AzızA

ar un beau jour de 1960, les spectateurs qui se hâtaient pour voir le dernier film de Georgio Ferroni, Les Bacchantes, ne se doutaient pas que le futur maître du péplum épique, le chantre d'Énée (La Guerre de Troie, 1961), de Mucius Scaevola (Le Colosse de Rome, 1961), de Coriolan (La Terreur des gladiateurs, 1964) allait profondément les traumatiser. Ferroni venait d'être reconnu comme l'un des maîtres du film d'horreur et tous les amateurs avaient salué son Moulin des supplices, 1959. Nul doute qu'avec Les Bacchantes, il allait rééditer, dans le genre antique, de si délicieux frissons.

Les malheureux furent détrompés très vite. Dès le générique, une voix off leur apprit l'étendue de leur infortune. Voici quelles étaient les paroles obscures qui s'écoulaient de sa bouche :

Ô bienheureux celui qui, par une faveur du Destin, est initié au mystère des dieux! Il sanctifie sa vie : le thiase exalte son âme sur les montagnes où il célèbre Bacchus par de saintes purifications! Heureux celui qui célèbre les orgies de Cybèle la déesse, selon la loi divine! Heureux celui qui, brandissant le thyrse, couronné de lierre, sert Dionysos! Allez, Bacchantes, allez, Bacchantes! Bromios, fils de dieu, emmène-les des montagnes de Phrygie aux cités florissantes de la Grèce.

Dans le silence angoissé qui suivit, seuls deux lycéens, dont les noms nous sont, hélas, restés inconnus, comprirent qu'ils avaient été les victimes d'un destin vengeur : c'est en vain qu'ils avaient séché leurs cours de grec, ils étaient en présence d'un texte traduit, dans lequel ils reconnurent sans plus tarder les 
accents chevrotants d'Euripide. En un éclair, ils virent la vérité dans toute son horreur : ces Bacchantes, c'étaient celle de la tragédie grecque du même nom. Et le texte qu'ils venaient d'entendre, c'était celui du chœur, encourageant, comme toujours, et à qui on n'avait pas laissé le temps de déclarer que c'était foutu, que celui-là qui avait atteint les portes de l'ultime jour pourrait - et encore avec des réserves - avoir été dit heureux qui comme Ulysse, etc. etc. Bref un chœur qui bêlait (ou qui bélier pour Ulysse), ajoutant à la tristesse ambiante qui, déjà, suintait du film.

Seuls les témoins de cette tragédie en chambre noire pourraient nous dire quels fructueux bénéfices les deux jeunes hellénistes retirèrent, en traduisant, séance tenante et suivante, le texte en langue vernaculaire plus accessible.

Cette traduction, nous l'avons retrouvée, la voici :

Il en a une sacrée chance, le mec qui, grâce à la roue de la fortune, est mis au parfum. On peut dire qu'il est peinard, il s'éclate avec la bande à Bacchus aux sports d'hiver où il se pinte tous les soirs, et, en plus, c'est gratuit. Même chose pour celui qui s'envoie en l'air dans les clubs pour couples libérés. Et pour celui qui, le walkman sur la tête, touche à son pote Dionysos. Caltez, Bacchantes ! Et toi, Bacchus, refile-les sans bromure (sans doute un contresens sur Bromios, qui est un autre nom du dieu Bacchus) du Club Méd aux discothèques.

Que s'était-il donc passé ? Ferroni connaissait vaguement la tragédie du malheureux Penthée qui, voulant s'opposer au culte de Bacchus, né de Sémélé, elle-même fille de Cadmos, roi de Thèbes, s'était vu lacéré par les Bacchantes, ivres de vin et d'orgueil, dont sa propre mère, Agavé, trop pleine de la nourriture divine. Mais, trouvant l'intrigue vraiment trop simplette, il lui avait rajouté d'autres mythes thébains : celui de la sécheresse qui oblige Athamas à sacrifier à Zeus ses enfants Phrixos et Hellé (cycle de la Toison d'or), celui de Tirésias et de sa fille Mantô (cycle d'Edipe), celui d'Actéon, enfin, dévoré par ses propres chiens, pour avoir surpris Diane au bain.

D'où un ensemble d'une extrême luxuriance, où éclate la personnalité de Bacchus, sobrement interprété par Pierre Winnetou-Brice et voluptueusement égayé par des Bacchantes en folie, dont l'unique plaisir semble de battre le tamtam, dans un réflexe onaniste qui en dit long sur leurs frustrations. Il faudra, pour réveiller le spectateur, qui est déjà dans un coma profond, que les Néo-Bacchantes thébaines, ivres de vin et de luxure, se livrent à quelques-unes de ces danses si lascives, dont le péplum raffole et qu'on enseigne, depuis un siècle, dans tous les couvents des oiseaux. 
Moralité, morale mythée : des Bacchanales aux bac-annales à chacun sa vérité. Un zér/eau pinté ou un vin/gt sur vin/gt.

\section{Claude Aziza}

Université de la Sorbonne Nouvelle, Paris III

claude.aziza@laposte.net 
